Received: 2021/06/12, Revised: 2021/06/29 Accepted: 2021/06/29, Published: 2021/06/30 (๑2021 Satoshi Hattori et al.; Licence Physical Activity and Nutrition. This is an open access article distributed under the terms of the creative commons attribution license (https:ll creativecommons.org/licenses/by-nc/2.0/), which permits unrestricted use, distribution, and reproduction in any medium, provided the orginal work is properly cited.

${ }^{*}$ Corresponding author : Naomi Omi, Ph.D.

Faculty of Health and Sport Sciences, University of Tsukuba, 1-1-1 Tennodai, Tsukuba, Ibaraki, 305-8574, Japan

Tel: +81-29-853-6319 / Fax: +81-29-853-6507

E-mail: omi.naomi.gn@u.tsukuba.ac.jp

(๑2021 The Korean Society for Exercise Nutrition

\section{The effects of royal jelly protein on bone mineral density and strength in ovariectomized female rats}

\author{
Satoshi Hattori ${ }^{1, \S} /$ Naomi Omi $1,{ }^{1},{ }^{*}$ \\ 1. Faculty of Health and Sport Sciences, University of Tsukuba, Tsukuba, Japan \\ $\S$. These authors contributed equally to this work
}

[Purpose] Sex hormones deficiency leads to dramatically bone loss in particular postmenopausal women. Royal jelly has anti-osteoporosis effect due to maintain bone volume in that condition. We hypothesized that royal jelly protein (RJP, a latent residue after extracting royal jelly) also prevents bone deficient in ovariectomized (OVX) female rats, the animal model of postmenopausal women.

[Methods] Female Sprague-Dawley rats $(n=30,6$ weeks age old) were sham operated (Sham; sham operated group, $n=7$ ), OVX control group (OC, $n=$ $7)$, OVX with low RJP intake group (ORL, $n=8)$, and $\mathrm{OVX}$ with high RJP intake group $(\mathrm{ORH}, \mathrm{n}=8)$ during 8 weeks experimental periods. In the end point of this experiment, the bone samples (lumbar spine, tibia, and femur) were surgically removed under anesthesia.

These bone samples were evaluated bone mineral density (BMD) and bone strength.

[Results] BMD of lumbar spine in RJP intake groups (ORL, ORH) were higher than that in OC group $(\mathrm{p}<0.05$ and $p<0.01$ ) in RJP intake volume dependent manner. BMD of tibial proximal metaphysis and diaphysis in RJP intake groups were also higher than these in OC group $(p<0.01$ and $p<0.01 / p<0.05$ and $p<0.001$ ) In addition, breaking force of femur in RJP intake groups were significantly increase compared with that in OC group ( $p<0.001$ respectively).

[Conclusion] These findings indicate that RJP contribute to prevent sex hormone related bone abnormality.

[Key words] royal jelly protein, BMD, bone strength, OVX, osteoporosis, postmenopausal women

\section{INTRODUCTION}

Bone is responsible organ for physical function (e.g. support, exercise, and protection) while changing its morphology due to bone resorption and bone formation. The dynamically metabolism by osteoclast and osteoblast is named "bone remodeling". The imbalance of bone remodeling leads to the development of progressive osteopenia with consequent pain and functional limitations'. Various nutritional interventions were studied to reduce fracture risk and prevent bone $\operatorname{loss}^{2}$. On the other hand, it still being discussed whether supplements of nutrients can improve bone health remains unknown for most nutrients.

Osteoporosis is a systemic skeletal disorder characterized by low bone mass, micro-architectural deterioration, and bone strength deterioration. This phenomenon accompanied with abnormal bone remodeling (excessive bone resorption compared with bone formation) is particularly developed in postmenopausal women ${ }^{3}$. On the other hand, various pharmacological therapies with novel mechanisms were considered to reduce the risk of fractures (e.g. raloxifene, teriparatide, and dehydroepiandrosterone $\left.{ }^{4,5}\right)$. However, some medications have suspected influence on cancer risk, heart attack, and stroke ${ }^{6}$. Thus, it is required a new therapeutic nutritional supplements for management and promotion of bone health.

Phytochemicals with preventive effects against bone deteriorations have recently received increasing attention since they are potentially more suitable for long-term use than traditional therapeutic chemical compounds $^{2}$. Royal jelly (RJ), a product secreted by young honeybee workers has several physiological activities such as anti-hypertension, anti-tumor, and anti-microbial ${ }^{7,8}$. RJ prevents ovariectomy (OVX) induced bone loss through the inhibition of RANKL (receptor activator of nuclear factor-kB (NF-kB) ligand)-induced osteoclastogenesis ${ }^{9}$. In contrast, royal jelly protein (RJP) is a latest source of fresh royal jelly ${ }^{10}$. It may have possibilities such as osteoblast activation ${ }^{11}$ and osteoclast differentiation through attenuating the induction of nuclear factor of activated $\mathrm{T}$ cells (NFAT) cl (a key transcription factor for osteoclast differentiation) ${ }^{9}$ like a RJ ingestion. Thus, RJP may also have the beneficial effects of bone 
health, whereas the effectiveness is not well known. If RJP can prevent bone fragility, the latent residue may be used as a food material with the similarly effect as RJ.

The purpose of this present study is to investigate the effect of RJP on bone properties (bone mineral density and strength) in OVX rats. We hypothesized that RJP also inhibits bone deterioration in these animal models. If we reveal that RJP prevent OVX-induced bone loss, the results contribute to nutritional treatment for postmenopausal osteoporosis.

\section{METHODS}

\section{Experimental design}

Schematic diagram of the experimental design is shown in Figure 1. Female Sprague-Dawley rats $(n=30,6$ weeks age old) were purchased from CLEA Japan (CE-2, CLEA Japan, Inc., Japan). They were preliminary breeding during 6 days, and they were surgically sham operated (Sham; sham operated group, $\mathrm{n}=7$ ) or ovariectomized $(\mathrm{n}=23)$ based on our previous study ${ }^{5}$. OVX group were randomized into three groups: OVX Control group (OC, $\mathrm{n}=7$ ), OVX with low RJP intake group (ORL, $\mathrm{n}=8$ ), and OVX with high RJP intake group $(\mathrm{ORH}, \mathrm{n}=8)$. Other protein component is casein. These rats were fed a low calcium diets $(0.3 \%$ calcium, $0.3 \%$ phosphate) during the 8 weeks experimental periods. RJP were added on this diet (ORL group; $0.48 \%$ / protein $(0.48 \mathrm{~g}$ RJP / 100g diet), ORH group; $30 \%$ / protein $(5.4 \mathrm{~g}$ RJP / 100g diet)) after 3 weeks. These rats were housed in individual single cages, and they could freely access to food and distilled water in ad-libitum. Food intake and body weight gain were measured every second day. The room was maintained at $23 \pm 1{ }^{\circ} \mathrm{C}$ (humidity, $50 \pm$ $5 \%$ ) under a $12: 12 \mathrm{~h}$ light-dark cycle (light 8:00 to 20:00).
Animal care and experimental procedures were approved by the Experimental Animal Committee of the University of Tsukuba. All animal groups were fasted in the day before the dissection. The femurs, tibias, and lumber spine were collected after euthanasia, and these samples were stored in $70 \%$ ethanol after cleaned of soft tissue. Animal care and experimental procedures were approved by the Experimental Animal Committee of the University of Tsukuba (07038).

\section{BMD measurement}

BMD of the tibias and lumber spine were evaluated dual energy X-ray absorptiometry (DXA; Aloka, DCS-600R, Tokyo, Japan) referenced as previous study ${ }^{12}$. Briefly, the tibias were separated into five divisions. The first division was considered proximal metaphysis site, it mainly composed as the cortical bone. And, the second to third divisions were regarded the diaphysis site.

\section{Bone strength testing}

The bone strength of the femoral midshafts were evaluated by a three-point bending test (DYN-1255; IIO DENKI, Tokyo, Japan) using previously described methods (distance between the fulcrum $1 \mathrm{~cm}$, plunger speed $100 \mathrm{~mm} /$ minute, full scale $50 \mathrm{~kg}$, chart speed $120 \mathrm{~cm} / \mathrm{min})^{13}$. "Breaking force" refers to the loading weight (gravitational acceleration) required for bone breaking. (workload was moved when a power of 1 dyn was applied).

\section{Statistics}

All data were expressed as the mean \pm standard error (SE) and were analyzed with SPSS (version 18.0 J; SPSS Inc., Chicago, IL). One-way analysis of variance (ANOVA) was used to test for statistically significant differences among groups. If a significant difference was detected among

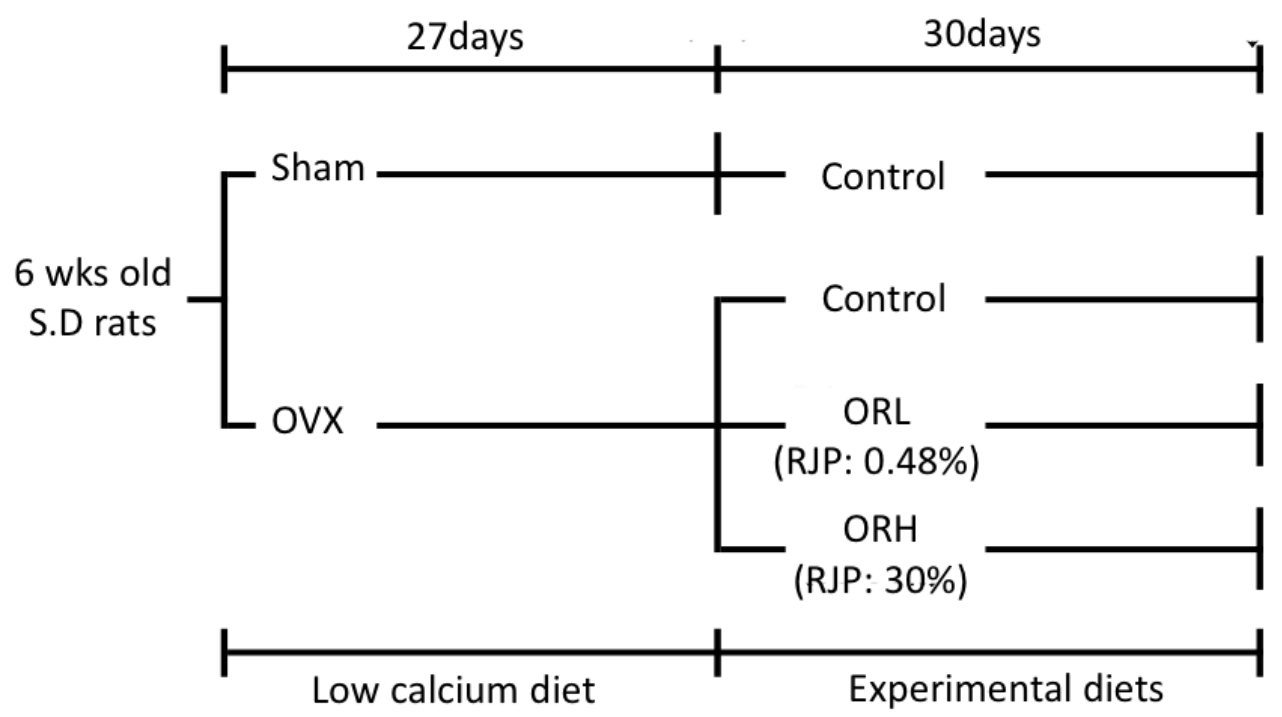

Figure 1. Schematic diagram of the experimental design. Female Sprague-Dawley (S.D.) rats were divided Sham (sham operated group, $n=7$ ), OVX Control group (OC, $n=7)$, OVX with low RJP (royal jelly protein) intake group (ORL, $n=8)$, and OVX with high RJP intake group (ORH, $n=8)$. 
groups, these groups were further evaluated using the Fisher's F-test for multiple comparisons. The significance level for major effects was set at $\mathrm{p}<0.05$.

\section{RESULTS}

Final body weight, body weight gain, food intake, and food efficiency are shown in Table 1. These parameters in OC group were significantly increased than these in sham group $(\mathrm{p}<0.001)$.

Table 1. Final body weight, Body weight gain, food intake, and food efficiency after experiment periods.

$\begin{array}{ccccc} & \begin{array}{c}\text { Final body } \\ \text { weight }(\mathrm{g})\end{array} & \begin{array}{c}\text { Body weight } \\ \text { gain (g/day) }\end{array} & \begin{array}{c}\text { Food intake } \\ \text { (g/day) }\end{array} & \begin{array}{c}\text { Food } \\ \text { efficiency }\end{array} \\ \text { Sham } & 274.9 \pm 7.5 & 1.56 \pm 0.08 & 15.42 \pm 0.49 & 0.10 \pm 0.00 \\ \text { OC } & 390.3 \pm 9.9^{* * *} & 2.95 \pm 0.16^{* * *} & 19.69 \pm 0.48^{* * *} & 0.15 \pm 0.01^{* * *} \\ \text { ORL } & 400.0 \pm 8.2 & 3.30 \pm 0.16 & 20.40 \pm 0.56 & 0.16 \pm 0.00 \\ \text { ORH } & 393.6 \pm 11.6 & 3.09 \pm 0.23 & 20.30 \pm 0.69 & 0.15 \pm 0.01\end{array}$

Values are mean \pm SE. ${ }^{a}$ Food efficiency was calculated by "body weight gain / food intake". " $p<0.001$ vs sham group.

There were not significantly differences the BMC of lumbar spine, BMC of tibial proximal metaphysis between these groups (Table 2). On the other hand, BMC of tibial diaphysis in OVX group (OC, ORL, ORH group) were higher than that in sham group $(\mathrm{p}<0.01, \mathrm{p}<0.01, \mathrm{p}<0.001$, respectively, Table 2).

Table 2. BMC of lumbar spine, BMC of tibial proximal metaphysis, and BMC of tibial diaphysis.

$\begin{array}{cccc}\text { lumbar spine (g) } & \begin{array}{c}\text { BMC of } \\ \text { proximal } \\ \text { metaphysis }(\mathrm{g})\end{array} & \begin{array}{c}\text { BMC of tibial } \\ \text { diaphysis }(\mathrm{g})\end{array} \\ \text { Sham } & 0.238 \pm 0.009 & 0.102 \pm 0.003 & 0.099 \pm 0.118 \\ \text { OC } & 0.234 \pm 0.005 & 0.097 \pm 0.003 & 0.114 \pm 0.002^{* *} \\ \text { ORL } & 0.233 \pm 0.004 & 0.098 \pm 0.003 & 0.114 \pm 0.003^{* *} \\ \text { ORH } & 0.236 \pm 0.004 & 0.100 \pm 0.003 & 0.120 \pm 0.003^{* * *}\end{array}$

Values are mean $\pm S E$. ${ }^{a}$ Food efficiency was calculated by "body weight gain / food intake". " $p<0.001$ vs sham group.

The BMD of lumber spine and tibia at proximal metaphysis in OC group was significantly decrease compared with these in sham group ( $p<0.01$, Figure $2 A-B)$, whereas that at diaphysis in $\mathrm{OC}$ group was higher than that in sham group ( $\mathrm{p}<0.05$, Figure $2 \mathrm{C}$ ).

On the other hand, the BMD of these bone parts in ORL and ORH groups were significantly increase these in sham group in a RJP concentration-dependent manner $(\mathrm{p}<0.05$, Figure 2A-C).

The breaking force of femurs in OC group was higher than that in sham group $(\mathrm{p}<0.001$, Figure 3$)$, these in ORL and ORH groups were also significantly increase these in sham group $(\mathrm{p}<0.001$, Figure 3$)$.

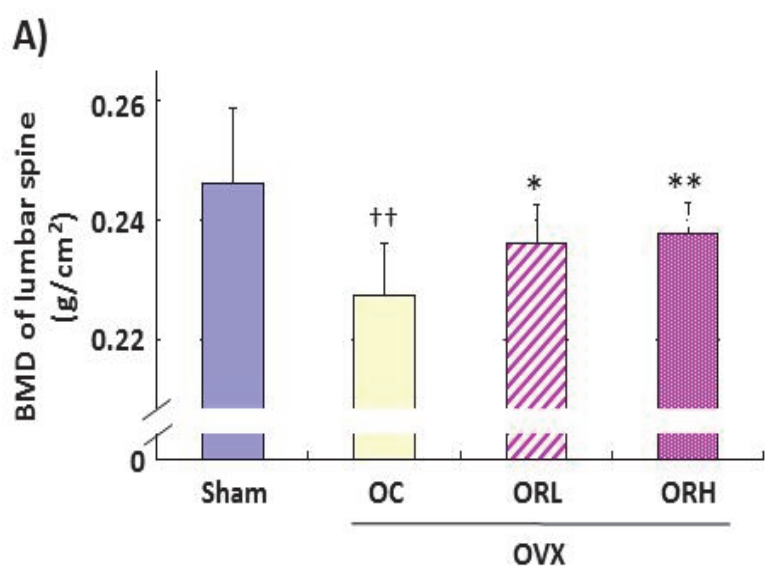

B)
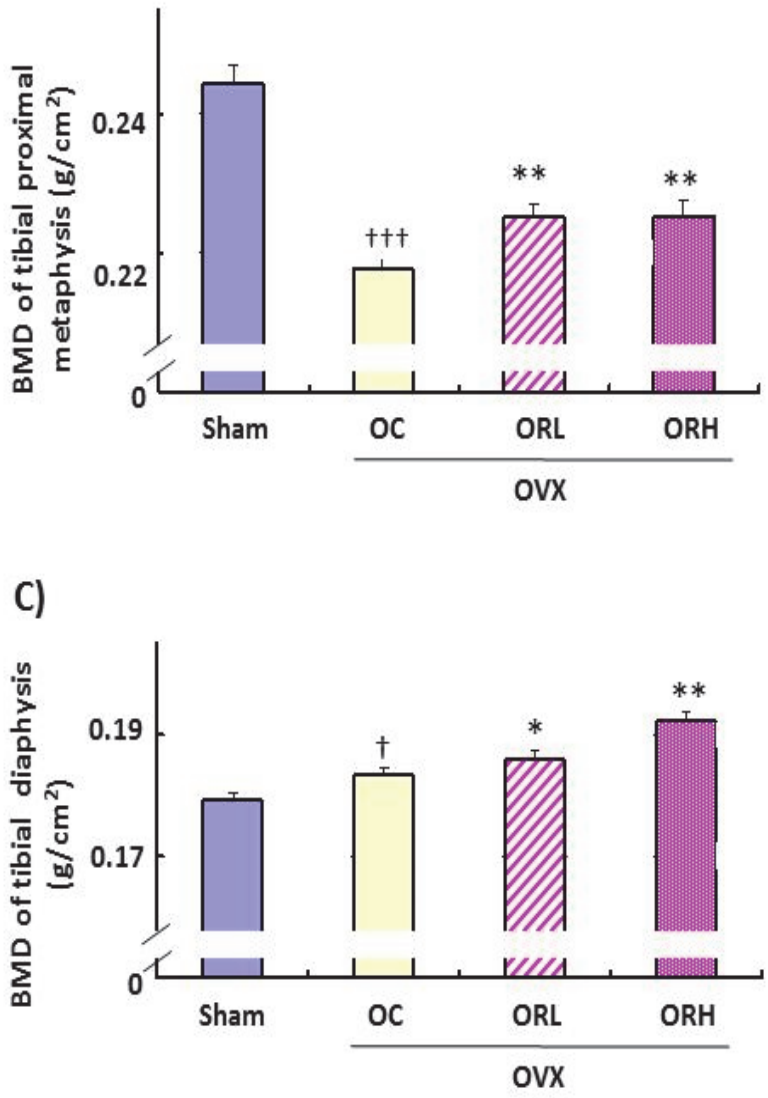

Figure 2. BMD of lumbar spine (A), BMD of tibia at proximal metaphysis (B), and that at tibial diaphysis (C). BMD levels were measured by DXA. Sham (sham operated group, $n=7$ ), OVX Control group (OC, $n=7$ ), OVX with low RJP (royal jelly protein) intake group (ORL, $n=8)$, and OVX with high RJP intake group (ORH, $n$ $=8)$. Data show mean $\pm \mathrm{SE}$.

\section{DISCUSSION}

Osteoporosis is a bone disorder that increases fracture risk in particular post-menopausal women. Various nutritional intervention trials are debated to reduce fracture risk $^{2}$. RJ is one of the effective treatments since it has diverse physiological functions such as improvement of bone 


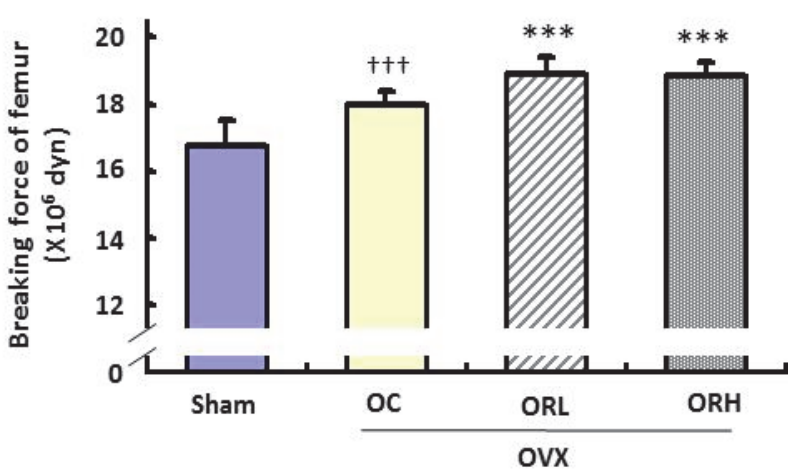

Figure 3. Breaking force of femur. Breaking force was measured by three-point bending test. Sham (sham operated group, $n=7$ ) OVX Control group (OC, $n=7$ ), OVX with low RJP (royal jelly protein) intake group (ORL, $n=8$ ), and OVX with high RJP intake group $(\mathrm{ORH}, \mathrm{n}=8)$. Data show mean $\pm \mathrm{SE}$.

metabolism ${ }^{14}$. However, it was not well known whether a latent residue of RJ (RJP) also has anti-osteoporosis effects. Thus, we investigated that RJP inhibits OVX induced bone loss using postmenopausal women model rats. As present results, we confirmed RJP suppressed decrease in BMD and bone strength according to OVX. These results indicate that RJP prevent bone abnormality caused by sex hormonal deficiency.

It is well known that OVX induce dramatically trabecular bone loss due to imbalance of bone remodeling ${ }^{15,16}$. In contrast, RJ inhibits the architectural breakdown of trabecular bone due to suppressing the differentiation of osteoclasts under sex hormonal deficient condition ${ }^{9}$. Moreover, RJP enhance a proliferation of osteoblast-like MC3T3-E1 and increase bone ash contents in animal model ${ }^{14}$. We also observed the RJP prevents a decreasing BMD levels at lumber spine and proximal tibia in OVX group. These findings indicate that RJP is possible to suppress trabecular bone loss in OVX rats.

Breaking force of femur in OC group is higher than Sham group, and RJP intake groups (ORL, ORH) is even higher in RJP concentration-dependent manner. Bone strength is determined not only by total of hydroxyapatite volume but also by collagen crosslink ${ }^{17}$. Actually, bone fragility is strongly related with collagen metabolism in post-menopausal women ${ }^{18}$. In contrast, it is reported that RJ could contribute bone quality due to modulating the posttranslational modification of type I collagen ${ }^{11}$. Then, RJP might involve bone strength via other factor of bone volume such as collagen metabolism, but that possibility needs further consideration.

BMD of tibial diaphysis (mainly composed by cortical bone) may be more susceptible than that of proximal metaphysis (mainly composed by trabecular bone) due to mechanical-loading ${ }^{19}$. The results of this study observed BMD of tibial diaphysis in OC group was higher than that in Sham group despite the opposite result in BMD of tibial proximal metaphysis. Similar trends have been observed in our previous studies that also used OVX rats ${ }^{13}$. These findings indicate that tibial diaphysis may be more sensitive than proximal metaphysis.

This study has two limitations to investigate the relationship of RJP and bone properties in OVX rats. First, the physiological basis of RJP was not evaluated, in particular bone remodeling. Then, it is unknown why OVX induced bone loss was suppressed and whether the phenomenon are based on a mechanism similar to previous study such as osteoclastgenesis ${ }^{9}$. This study needs to focus on the molecular biological approach in the future. Second, strict RJP intake was not controlled because this experiment was designed by ad-libitum intake to avoid the effects of food restriction. Hence, it is undeniable that the effect of RJP may have an error within the group. Third, the composition of RJP is not evaluated in detail. Therefore, it is unknown which content of RJP affected the bone.

In conclusion, we evaluated the effect of RJP on OVX induced bone abnormality. RJP inhibited decreased BMD and bone strength in various bone parts. These findings indicate RJP is useful to prevent sex hormone related bone fragility.

\section{ACKNOWLEDGEMENTS}

We would like to thank members of exercise and nutrition laboratory in University of Tsukuba for useful discussions and supports.

\section{REFERENCES}

1. Langdahl B, Ferrari S, Dempster DW. Bone modeling and remodeling: potential as therapeutic targets for the treatment of osteoporosis. Ther Adv Musculoskelet Dis. 2016;8:225-35.

2. Tabatabai LS, Sellmeyer DE. Nutritional supplements and skeletal health. Curr Osteoporos Rep. 2021;19:23-33.

3. Lerner UH. Bone remodeling in post-menopausal osteoporosis. $J$ Dent Res. 2006;85:584-95.

4. Bellavia D, Dimarco E, Costa V, Carina V, De Luca A, Raimondi L, Fini M, Gentile C, Caradonna F, Giavaresi G. Flavonoids in bone erosive diseases: perspectives in osteoporosis treatment. Trends Endocrinol Metab. 2021;32:76-94.

5. Hattori S, Park S, Park JH, Omi N. The effect of dehydroepiandrosterone administration on intestinal calcium absorption in ovariectomized female rats. Phys Act Nutr. 2020;24:24-7.

6. Chen LR, Ko NY, Chen KH. Medical treatment for osteoporosis: from molecular to clinical opinions. Int J Mol Sci. 2019;20:1-21.

7. Pasupuleti VR, Sammugam L, Ramesh N, Gan SH. Honey, propolis, and royal jelly: a comprehensive review of their biological actions and health benefits. Oxid Med Cell Longev. 2017;2017:1259510.

8. Cornara L, Biagi M, Xiao J, Burlando B. Therapeutic properties of bioactive compounds from different honeybee products. Front Pharmacol. 2017;8:412.

9. Tsuchiya Y, Hayashi M, Nagamatsu K, Ono T, Kamakura M, Iwata T, Nakashima T. The key royal jelly component 10-hydroxy-2-decenoic acid protects against bone loss by inhibiting NF-KB signaling downstream of FFAR4. J Biol Chem. 2020;295:12224-32.

10. Matsui T, Yukiyoshi A, Doi S, Sugimoto H, Yamada H, Matsumoto 
K. Gastrointestinal enzyme production of bioactive peptides from royal jelly protein and their antihypertensive ability in SHR. J Nutr Biochem. 2002;13:80-6.

11. Kaku M, Rocabado JMR, Kitami M, Ida T, Uoshima K. Royal jelly affects collagen crosslinking in bone of ovariectomized rats. $J$ Funct Foods. 2014;7:398-406.

12. Hattori S, Park JH, Agata U, Akimoto T, Oda M, Higano M, Aikawa Y, Nabekura Y, Yamato H, Ezawa I, Omi N. Influence of food restriction combined with voluntary running on bone morphology and strength in male rats. Calcif Tissue Int. 2013;93:540-48.

13. Hattori S, Agata U, Park J-H, limura Y, Tokuda S, Ezawa I, Omi $\mathrm{N}$. The relationship between salivary calcium concentration and differences in bone mineral density level in female rats. J Nutr Sci Vitaminol. 2014;60:152-8.

14. Narita Y, Nomura J, Ohta S, Inoh Y, Suzuki KM, Araki Y, Okada S, Matsumoto I, Isohama Y, Abe K, Miyata T, Mishima S. Royal jelly stimulates bone formation: physiologic and nutrigenomic studies with mice and cell lines. Biosci Biotechnol Biochem. 2006;70:250814.

15. Wronski TJ, Dann LM, Horner SL. Time course of vertebral osteopenia in ovariectomized rats. Bone. 1989;10:295-301.

16. Wronski TJ, Walsh CC, Ignaszewski LA. Histologic evidence for osteopenia and increased bone turnover in ovariectomized rats. Bone. 1986;7:119-23.

17. Garnero P. The contribution of collagen crosslinks to bone strength. Bonekey Rep. 2012;1:182.

18. Gertz BJ, Shao P, Hanson DA, Quan H, Harris ST, Genant HK Chesnut $\mathrm{CH}$ 3rd, Eyre DR. Monitoring bone resorption in early postmenopausal women by an immunoassay for cross-linked collagen peptides in urine. J Bone Miner Res. 1994:9:135-42.

19. Ott SM. Cortical or trabecular bone: what's the difference? Am J Nephrol. 2018;47:373-5. 